\title{
Guest editorial Rethinking international manufacturing in times of global turbulence
}

\section{The need of rethinking international manufacturing in times of global turbulence}

Indisputably, the trend of international manufacturing has been bound to persist and grow (Cheng et al., 2014). With the prevalence of globally distributed plants and suppliers, international manufacturing has already been a complex issue for both practitioners and researchers. Notwithstanding, in recent years, a series of shifts in the global economic and institutional environments have made firms reconsider their internationalisation strategies. Not only emerging markets have become important but new competitors from those countries also challenging incumbent structures. Trade wars, especially between East and West and between the USA and China, associated with conservative winds and national protectionism, have been fueling a resurgence. A growing support for policy interventions to reverse internationalisation or de-globalisation has also led to initiatives, adopted by the US and the Japanese governments, for attracting multinational enterprises (MNEs) to backshore their offshored manufacturing activities. This process is further expected to be supported by the introduction of digital technologies (such as big data analysis, artificial intelligence, 3D print, etc.). These have started to change the traditional production methods for many products with profound implications for product-service offerings, manufacturing location and supply chain design. In addition, the constant emphasis on environmental protection is also believed to have a significant influence on international manufacturing. As a response to the recent initiatives of many countries regarding carbon neutrality, manufacturing companies will have to reconsider their international manufacturing setups. Last but not least, the COVID-19 pandemic has inevitably brought tremendous challenges to manufacturing companies. With such a variety of global turbulence, a discussion of current trends and future trajectories is both timely and necessary (Brennan et al., 2015). Thus, this special issue aims at rethinking international manufacturing in times of global turbulence.

\section{Selected papers in this special issue: what do they reveal?}

Two major research tracks can be distinguished regarding international manufacturing, namely manufacturing network research and supply chain research (Rudberg and Olhager, 2003). The former is defined as a coordinated aggregation (network) of intra-firm manufacturing facilities located in different places and as an internal network with all facilities under full financial control (Cheng et al., 2014), while the latter is analysed as an external (inter-firm) network with manufacturing facilities owned by different organisations and characterised by sets of purposeful and interconnected exchange relationships to transform resources into finished products for end customers. The 11 papers selected for this special issue cover issues within or between the two research tracks.

First, there are three papers that solely address manufacturing networks, namely Arellano et al. (2021), Deflorin et al. (2021) and Wiech and Friedli (2021). Among them, Arellano et al.

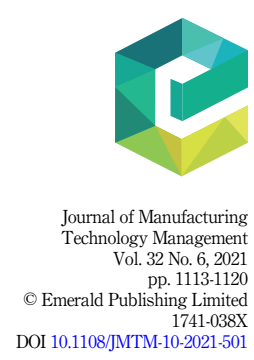


JMTM 32,6

1114

(2021) and Wiech and Friedli (2021) suggest the increased relevance of intangible dimensions to maintain the integrity of the international manufacturing network in times of turbulence. Specifically, Arellano et al. (2021) focus on plants within a manufacturing network and investigate the relationship between different dimensions of culture and manufacturing integration. Likewise, Wiech and Friedli (2021) also focus on plants within a manufacturing network, but they pay more attention to plant leaders and attempt to identify barriers to intranetwork exchange within the manufacturing network from plant leaders' perspective. Unlike these studies, Deflorin et al. (2021) analyse the relation between industrial Internet of things (IoT) and manufacturing network coordination.

Second, three papers focus on supply chains, namely Basu et al. (2021), Saglam et al. (2021) and Veile $e t$ al. (2021). The first two emphasise the need to follow an evolutionary process to create the responsiveness required for operations in VUCA (volatile, uncertain, complex and ambiguous) types of markets. Specifically, Basu et al. (2021) explore the mediation effect of volatile economic conditions on performance benefits of successful lean manufacturing implementation for plants in the context of the supply chain. Saglam et al. (2021) explore the relationship between significant proactive risk mitigation strategies (namely, supply chain flexibility, resilience and responsiveness) and supply chain risk management performance, as well as the moderating role of risk management culture on such relationship. On the other hand, the third paper, Veile et al. (2021) focus on the new features of buyer-supplier relationships in times of digitalisation and analyse how technological changes in the context of industry 4.0 influence the buyer-supplier relationship.

Third, four papers attempt to discuss manufacturing network and supply chain simultaneously, namely Cheng et al. (2021), Das and Dey (2021), de Borja Reis (2021) and Thakur-Weigold (2021). Among them, Das and Dey (2021), de Borja Reis (2021) and ThakurWeigold (2021) indicate the need to rethink and redefine international manufacturing operations when firms change their business model to embrace servitisation/digitalisation or relocate to focus on preferential markets. Specifically, Das and Dey (2021) aim to synthesise a manufacturing value network for digital multinationals that combines the global reach of multinationals, the power of platform business models and digitalisation. Similarly, de Borja Reis (2021) combines global value chains, international manufacturing networks and cost management literature to investigate if the world top manufacturing corporations' cost structures are moving from tangible to intangible activities, in other words, increasing the share of services in their portfolios. Furthermore, Thakur-Weigold (2021) explores the restructuring of a contract manufacturer operating under the shifting conditions of a global virtual manufacturing network. Different from these studies, Cheng et al. (2021) investigate the moderating effect of the role a plant play in a manufacturing network on the relationships between its level of integration with other plants in the same manufacturing network, its interactions with suppliers/customers and its operational performance.

Fourth and last, Engelseth et al. (2021) pay attention to global engineering services. They detect how to manage economisation of the maintenance and modification operations in offshore petroleum logistics operations and reveal the dynamics of building network capabilities in a consistent network structure.

\section{Moving towards the future}

The papers selected for this special issue contribute to rethinking international manufacturing in times of global turbulence. However, they only represent limited research on this topic. There are still a lot to do. Inspired by them, further potential research opportunities are suggested.

\subsection{A total cost perspective for plant location advantage}

Five papers in this special issue pay attention to plants embedded in either a manufacturing network or a supply chain. In fact, manufacturing plants can be viewed as the integral parts 
and even the basic constructs of a manufacturing network and a supply chain/network (Cheng et al., 2014). Their roles are normally determined according to two dimensions, i.e. location advantages and site competences (Ferdows, 1997). Regarding the former, the existing research always considers access to low-cost production, proximity to market and access to skills and knowledge, as the three main location advantages (e.g. Vereecke and Van Dierdonck, 2002; Cheng and Farooq, 2018 as well as Cheng et al., 2021 in this special issue). However, along with the development of manufacturing technologies, access to low-cost production seems to be not as important as before. It is possible for more American and European MNEs to move some of their production activities back to home countries, where labour costs are even higher. All depend on the calculations of total costs rather than individual types of costs. Similarly, companies can benefit from moving their production in China to other Southeast Asian countries, like Vietnam and India, only when they consider the total costs behind rather than merely chasing low-cost production resource. Due to poor infrastructure and less efficient and qualified workforce, it is always not cheaper to produce in these countries in the short term. Not to mention production in these countries might still need to procure from China, and their products might still need to be sold in China, as China has the most complete supply chain systems and the biggest market. Thus, it is time to reconsider the cost aspect of plant location by moving away from low-cost production to a total cost perspective.

\subsection{A holistic view on manufacturing network and supply chain}

To conduct total cost calculations, it is important to address what manufacturing processes of what products for what markets are to be allocated to what (either intra- or inter-firm) plants in what countries. Following this logic, a manufacturing network and a supply chain can be respectively viewed as an intra- or inter-firm pool with manufacturing resources for product and process allocations. This similarity opens the possibility of adopting a holistic view on manufacturing networks and supply chains. In fact, although manufacturing networks and supply chains are based different concepts and focuses, the boundaries between them are gradually in a continuous state of flux, including the proliferation of various forms of intraand inter-firm collaboration (Coe et al., 2008). As the link between structure and capability becomes intertwined in highly networked manufacturing and supply systems with multiple choices on ownership, location and integration, it is believed that manufacturing network and supply chain cannot be managed separately. Instead, when discussing manufacturing network, the attention should be paid to not only the strategies, decisions and capabilities of plants in the manufacturing network but also to the workflows and capability shifts between the plants (Fredriksson et al., 2019). Similarly, supply chain research also needs to go beyond the analysis of the flows between the plants and their external suppliers and take the roles of these plants in the manufacturing network into account (Potter and Wilhelm, 2020). Such a holistic view on manufacturing networks and supply chains indeed brings tremendous complexity to both research and management, but it also opens new opportunities for discussions, as demonstrated by four of the papers in this special issue that simultaneously address manufacturing network and supply chain.

Furthermore, inspired by Engelseth et al. (2021), Das and Dey (2021), de Borja Reis (2021) and Thakur-Weigold (2021), the holistic view of manufacturing network and supply chain might need to be further extended to cover other functions or even the entire value chain. In many cases, the internationalisation of manufacturing is not an end, but a starting point followed by the internationalisation of other value chain activities (e.g. research and development [R\&D], service). Because of multiple systematic linkages between different functions, their internationalisation has to be examined simultaneously. Unfortunately, there is currently no research that offers a comprehensive framework for managing intra/inter-firm 
JMTM 32,6

multi-functional facilities/networks of geographically dispersed operations along the value chain.

\subsection{The influence of digitalisation on international manufacturing}

As demonstrated by two papers in this special issue (i.e. Deflorin et al., 2021 and Veile et al., 2021), the wide adoption of industry 4.0 technologies can bring profound implications for international manufacturing. Different scenarios have already been envisioned:

(1) First, the adoption of robots makes production less dependent on manual labour. Accordingly, it can be more beneficial for MNEs to relocate their production to countries close to the main markets, even if these are high labour cost countries.

(2) Second, the adoption of 3D printing makes it possible to only transfer product designs, rather than physical products. In doing so, transport costs can be significantly or even entirely reduced. Hence, a new type of supply chains arises, focusing on the supply of material granulates, and there is a need of securing safe data transfers.

(3) Third, digitalisation and digitisation require the ability to collect, handle and utilise large datasets. It is foreseen that the edifice for the holistic management of manufacturing network and supply chain can quickly grow too complex for manual planning. In this case, a system that adopts IoT, big data and artificial intelligence can support decision-making based on objective data and detailed analysis. Some mathematical models have already been developed by academia, but they, on the one hand, have not kept up with the increasing complexity; on the other hand, they have been less used by industry due to weak operability. In practice, many companies still rely on manual calculations, some simple (Excel) tools and a lot of "guestimation".

(4) Fourth, how to respond to the acceleration of digitalisation and digitisation increases turbulence derived from consumers' behaviour. In fact, the consumer behaviour creates a double-edged sword because of the strong dependency between the digital and the physical world. Digital commerce channels increase the consumer ability to shortcut existing established supply chains by ordering at online platforms, such as Alibaba and Wish. However, because of their lack of knowledge about existing supply chain structures, consumers hunt for low prices, and their ability of "clickbuy" will require new actions in international manufacturing in order to mitigate possible negative consequences.

To summarise, in order to systematically understand the influence of digitalisation on international manufacturing, more research is still needed to explore what technologies can be applied for what scenarios and how. Moreover, upon until now, the digital flows have been developed to support the physical flows, though the future might be that the physical flows follow the digital flows. Future research is thus needed on how international manufacturing and supply chains should build up this ability.

\subsection{The influence of sustainability policies on international manufacturing}

Similarly, due to their lack of supply chain knowledge and impulsive behaviour, the consumers move away from the environmental, social and ethical considerations by shortcutting the existing supply chains and at the same time pressure for better environmental, social and ethical practices. This behaviour among the consumers creates increase the development of environmental harmful supply chains as well as removing power 
from the large companies in terms of CSR (corporate social responsibility) work. Meanwhile, responding to radical climate changes in recent years, the governments of different countries have also launched policies, especially related to sustainability. For example, in recent years, many countries have begun the legislation process for carbon neutrality, which is believed to influence international manufacturing.

\subsection{An international political economy perspective on international manufacturing}

It is obvious that the rationale behind international manufacturing has been influenced significantly by international political and economic relations, especially in recent years. Since the Second World War, the Western developed countries, especially the USA, have been and still are the centre of the world's capital market and the engine of the world economy. The current political landscape of the world is largely defined by these countries. They also still occupy the top level of global value chains and are normally responsible for innovation, highend production and service. Other developing countries survive and operate under this given order. Some of them are responsible for low/middle-end manufacturing, while others are responsible for providing raw materials. However, such political landscape and distribution of global value chains are now challenged by the development of developing countries, especially China. The Chinese Belt and Road Initiative is a potential threat to the current international order, and the technological development achieved by China, like 5G communication, further makes the Western developed countries feel more pressure regarding their position in the global value chain. Moreover, along with the development of China, the Western developed countries have entered the rhythm of de-industrialisation and accordingly suffer from an unemployment problem. Without being timely addressed, the unemployment problem has caused various social problems and imbalances. These social problems and imbalances have triggered a political shift towards populism in these countries, which in turn have had consequences on economic policies. As a result, it will not be surprising to see more (political and economic) conflicts, like trade wars, between these countries and China, which are bringing tremendous influences to MNE (no matter which home countries they are from) international manufacturing setups. Hence, more research is needed to examine international manufacturing from an international political economy perspective.

\subsection{International manufacturing after COVID-19}

Undoubtedly, the COVID-19 pandemic has changed and will continuously influence the context for international manufacturing. First, the problems related to the production of antipandemic supplies, such as masks and vaccines, stimulate a rise of nationalist interests and governmental intervention through policies aimed at guaranteeing market supply in developed countries. However, there is a discrepancy among the general understanding of the production capability and capacity within these countries and the actual ability, as well as the time it takes to rebuild these abilities. Thus, these production problems are not only due to the lack of capacity but also capability that has gone lost due to the long-term lack of focus from institutions regarding the strategic importance of production capability and ability within borders. Besides, compared to the relocation of production activities, it is more difficult to move the entire supply chain back in a short time. As a result, relocating production back to developed countries is happening, but will take time and be a stepwise process. Second, the COVID-19 pandemic attracts more attention to the concept of resilience. In the past years, there has been an increased dependency on the supply chain abilities, capacities and flexibility due to the distribution/outsourcing of production activities across companies and worldwide. Unfortunately, pandemic control efforts have interrupted flows of finished goods and raw materials from suppliers' factories in one country to many parts of the world. 
JMTM 32,6

1118

It has become a huge challenge to keep global supply chains going while some parts of the supply chain have stopped operations. The situation is even worse, if companies centralise their purchasing for economies of scale and buy materials/components from only a few suppliers or if certain key materials/components are controlled by a few suppliers worldwide or available within limited markets. The present semi-conductor crisis, stopping production in the vehicle industry, is an example of the latter.

It has been more than one year now since the break-out of the pandemic. Although more and more people have been vaccinated, it is still difficult to predict when the pandemic will end. Facing the disruptions caused by the pandemic, it is imperative for companies to restructure their international manufacturing network, supply chains or even global value chains, in order to better respond to the uncertainty and be resilient to the disruptions. More research is accordingly needed.

\section{Concluding remarks}

Due to the global turbulence in terms of the development of industrial resources in different countries, international political and economic relations, the increased focus on environmental, social and ethical considerations, the technological and digital development and other unforeseen events, like COVID-19, the factors influencing international manufacturing are profoundly changing, and the pattern of international manufacturing is at the tipping point of a transformation process. It is already possible to see some companies moving their production out from the World Factory, i.e. China, to Eastern Europe or Southeast Asia. Nevertheless, this does not mean that they can completely leave China. On the one hand, in the past decades, China has developed a large-scale and well-established supply chain system with complete industrial categories and infrastructure, which can satisfy diverse demands in an efficient manner. Such a supply chain system might not exist in the new countries. Furthermore, no one can ignore the mature market and the innovation potential of China. Even it is possible to identify more alternatives to accommodate production with low (total) cost, the options can offer less in terms of market and innovation. Hence, it is our bold assumption that MNEs might cultivate their operations in three regions, i.e. Northern America, European Union and Asia-Pacific. These regions can be viewed as complete manufacturing ecosystems with respective innovation hubs, markets and production bases:

(1) Northern America: innovation hub - USA; market - USA and production base Mexico.

(2) European Union (EU): innovation hub - founding countries of EU; market - founding countries of EU and production base - newly joined, especially eastern European, countries.

(3) Asia-Pacific: innovation hub - Japan, Korea and China; market - Japan, Korea and China; and production base - China, Vietnam, etc.

It is highly likely that companies will gradually focus on these regions separately in the near future, in order to, on the one hand, avoid potential political risks and, on the other hand, reduce the length and increase the control of their supply/value chains. The transformation process has been initiated by both companies and governments in the past years and further speeded up by the recent COVID-19 pandemic. It is expected that after this transformation, the pattern of international manufacturing will become more localised, regionalised and resilient. Certainly, during the transformation, new issues to be studied are continuously emerging. There is still a lot of work to be done. 
Yang Cheng

Department of Materials and Production, Center for Industrial Production, Aalborg University, Aalborg, Denmark and School of Business Administration, Jiangxi University of Finance and Economics, Nanchang, China

Anna Fredriksson Department of Science and Technology, Linköping University, Linköping, Sweden, and Afonso Fleury Production Engineering, Universidade de São Paulo, Sao Paulo, Brazil

\section{References}

Arellano, M.C., Sancha, C., Netland, T. and Thomsen, C.G. (2021), "Manufacturing network integration and culture: an institution-based view", Journal of Manufacturing Technology Management, Vol. 32 No. 6, pp. 1121-1143.

Basu, P., Chatterjee, D., Ghosh, I. and Dan, P.K. (2021), "Lean manufacturing implementation and performance: the role of economic volatility in an emerging economy", Journal of Manufacturing Technology Management, Vol. 32 No. 6, pp. 1188-1223.

Brennan, L., Ferdows, K., Golini, R., Keegan, R., Kinkel, S., Srai, J.S. and Taylor, M. (2015), "Manufacturing in the world: where next?", International Journal of Operations and Production Management, Vol. 35 No. 9, pp. 1253-1274.

Cheng, Y. and Farooq, S. (2018), "The role of plants in manufacturing networks: a revisit and extension”, International Journal of Production Economics, Vol. 206, pp. 15-32.

Cheng, Y., Farooq, S. and Johansen, J. (2014), "Global operations: a review and outlook", International Operations Networks, pp. 161-179.

Cheng, Y., Farooq, S. and Jajja, M.S.S. (2021), "Does plant role moderate relationship between internal manufacturing network integration, external supply chain integration, operational performance in manufacturing network?”, Journal of Manufacturing Technology Management, Vol. 32 No. 6, pp. 1267-1289.

Coe, N.M., Dicken, P. and Hess, M. (2008), "Global production networks: realizing the potential", Journal of Economic Geography, Vol. 8 No. 3, pp. 271-295.

Das, A. and Dey, S. (2021), "Global manufacturing value networks: assessing the critical roles of platform ecosystems and Industry 4.0”, Journal of Manufacturing Technology Management, Vol. 32 No. 6, pp. 1290-1311.

de Borja Reis, C.F., de Souza, A.B., Araujo, E.C. and Blind, K. (2021), "Value chains of the world's top manufacturing corporations: moving from tangible to intangible activities?", Journal of Manufacturing Technology Management, Vol. 32 No. 6, pp. 1312-1334.

Deflorin, P., Scherrer, M. and Schillo, K. (2021), “The influence of IIoT on manufacturing network coordination”, Journal of Manufacturing Technology Management, Vol. 32 No. 6, pp. 1144-1166.

Engelseth, P., Törnroos, J.Å. and Zhang, Y. (2021), "Interdependency in coordinating networked maintenance and modification operations", Journal of Manufacturing Technology Management, Vol. 32 No. 6, pp. 1357-1375.

Ferdows, K. (1997), "Making the most of foreign factories", Harvard Business Review, Vol. 75, pp. 73-91.

Frederiksson, A., Malm, A. and Madsen, E.S. (2019), "Technology transfer as a part of the businessinter-organizational transfer strategies based on experiences of aircraft production", Journal of Global Operations and Strategic Sourcing, Vol. 12 No. 1, pp. 151-171. 
JMTM 32,6
Potter, A. and Wilhelm, M. (2020), "Exploring supplier-supplier innovations within the Toyota supply network: a supply network perspective", Journal of Operations Management, Vol. 66 Nos 7-8, pp. 797-819.

Rudberg, M. and Olhager, J. (2003), "Manufacturing networks and supply chains: an operations strategy perspective", Omega, Vol. 31 No. 1, pp. 29-39.

Saglam, Y.C., Çankaya, S.Y. and Sezen, B. (2021), "Proactive risk mitigation strategies and supply chain risk management performance: an empirical analysis for manufacturing firms in Turkey”, Journal of Manufacturing Technology Management, Vol. 32 No. 6, pp. 1224-1244.

Thakur-Weigold, B. (2021), "Capability mapping to improve manufacturing network performance: how a factory can target growth”, Journal of Manufacturing Technology Management, Vol. 32 No. 6, pp. 1335-1356.

Veile, J.W., Schmidt, M.C., Müller, J.M. and Voigt, K.I. (2021), "Relationship follows technology! How Industry 4.0 reshapes future buyer-supplier relationships", Journal of Manufacturing Technology Management, Vol. 32 No. 6, pp. 1245-1266.

Vereecke, A. and Van Dierdonck, R. (2002), "The strategic role of the plant: testing Ferdows's model", International Journal of Operations and Production Management, Vol. 22 No. 5, pp. 492-514.

Wiech, M. and Friedli, T. (2021), "Using plant leaders' perspectives to overcome barriers to inter-plant exchange", Journal of Manufacturing Technology Management, Vol. 32 No. 6, pp. 1167-1187. 